\title{
Some Features of Statistical Quantum Chromo- dynamics in Relativistic Heavy Ion Collisions
}

\author{
Mohammad Ayaz Ahmad ${ }^{1}$, Mir Hashim Rasool ${ }^{2}$, Jalal Hasan Baker ${ }^{1}$, Shafiq Ahmad ${ }^{3}$ \\ ${ }^{1}$ Physics Department, Faculty of Science, P.O. Box 741, University of Tabuk, 71491, Tabuk, Saudi Arabia, \\ ${ }^{2}$ Department of Physics, Islamic University of Science and Technology, Awantipora, 192122, J\&K, India
}

${ }^{3}$ Physics Department, Aligarh Muslim University, Aligarh, 202002, India

\begin{abstract}
An attempt has been made to discuss the classical topic of statistical physics so called the microscopic dynamics and derive the equilibrium thermodynamics which describes a macroscopic system. Here, we try to explain the microscopic theory is the term of quantum chromo-dynamics (QCD), and we want to obtain the thermodynamics of strongly interacting matter. Finally, at this point we notice that two basic features of what we're about to study - the equilibrium condition and the arbitrarily large volume of the systems in question - may restrict the applicability of the results to nuclear collisions.
\end{abstract}

Keywords:relativistic heavy ion collisions, deconfinement state of matter, quark gluon plasma (QGP).

\section{Introduction}

The primary goal of high-energy heavy ion collisions is to discover the fundamental forces, symmetries and the elementary particles in Nature. On the other hand, Particle physics is the science of the fundamental structure of matter, which leads to the study of the properties of subatomic particles and mechanism of their interactions. Its ultimate aim is to find a complete description of the elementary constituents of matter and of the forces acting between them. The final structure of elementary particle is found to consist of quarks for which no structure has been observed for them, so they are regarded as the point like particles. These results have been obtained by scattering experiments at higher and higher energies, as required to achieve information on smaller and smaller objects. Any isolated single free quarks have never been observed experimentally, and therefore it is conjectured that quarks are confined together with other quarks to form hadrons. The strong (color) force field between the quarks is intermediated by gluons, and inside the hadrons quark-antiquark pairs are formed as quantum fluctuations.

The interest in the study of high-energy nuclear matter has increased many folds due to the possibility of studying unstable states of nuclear matter under extreme condition of high energy density and high temperature. Physicists are very keen to see its outcomes as they expect that it would throw its flashes towards the evolution of the universe and deconfined state of freely interacting quarks and gluons known as quark-gluon plasma (QGP) [1-5], which is believed to have existed in the form of QGP for few microseconds after the Big Bang. It is also interesting to study about the strong forces present between the quarks and gluons in the hadronic matters. It is believed that shortly after the creation of the Big Bang all matters were in a state called the QGP. Due to rapid expansion of the universe, this plasma went through a phase transition to form large number of hadrons like pions, protons and neutrons etc. Such a new phase of matter might be produced experimentally in heavy ion collisions at ultra-relativistic energies.

\section{Mathematical Descriptions}

The Quantum Chromo-Dynamics (QCD) [6-8] theory describes the strong interaction between the hadronic matter which predicts a novel phase transition from a confined state of quark and gluon to deconfined state, where quarks and gluons would be free to move and freely interacting in the hadronic matter, which would be at higher energy density.

The main emphasis of the present research work is to consider the classical topic of statistical physics, which give the microscopic dynamics, and also originate the thermodynamical equilibrium which defines a macroscopic system. Here in this case the microscopic theory is known as QCD, and we want to acquire the thermo-dynamical system of the strongly interacting matter. For this task we noted that there are two basic features of what we were studied; the equilibrium conditions and the arbitrarily large volume of the systems that might be restrict the applicability of the results to nuclear collisions.

The QCD theory assumed that the collisions between the colored quark and gluons [9] can be treated by the following Lagrangian density equation:

$$
\mathcal{L}(\Psi, \bar{\Psi}, \mathrm{A})=-\frac{1}{4}\left(\partial_{\mu} A_{v}^{\mathrm{a}}-\partial_{v} A_{\mu}^{a}-\mathbf{g f}_{\mathrm{b} \mathrm{c}}^{\mathrm{a}} A_{\mu}^{\mathrm{b}} A_{v}^{\mathrm{c}}\right)^{2}+\bar{\Psi}_{\mathrm{f}}^{\mathrm{a}}\left(\mathrm{i} \partial-\mathrm{g} \lambda_{\mathrm{a}} \lambda_{\alpha \beta}^{\mathrm{a}}\right) \Psi_{\mathrm{f}}^{\beta}(1)
$$

where; $\psi$ and A represent to the quark and gluon fields, with equivalent color indices $\alpha, \beta, \ldots$. and $a, b, \ldots \ldots$ and " $\mathrm{f}$ " indicates the flavor of quarks (i.e. $\mathrm{u}, \mathrm{d}, \mathrm{s} .$. ) and the " $\mathrm{g}$ " is the dimensionless coupling factor of the theory. The first term on the right hand side (R.H.S.) of Equation (1) explains the interaction of gluons; this interaction is possible - in contrastto the quantum electrodynamics (QED), where the photons do not interact directly - because the gluons also carrya color charge. The second term of Equation (1) is for the interactions of quarks and gluons, and this is basically the same structure as its electromagnetic counterpart. The specificfeature of QCD is thus the interaction between gauge fields, and one may studypure gauge field thermodynamics as a meaningful model of statistical QCD. 


\section{International Journal of Science and Research (IJSR) \\ ISSN (Online): 2319-7064 \\ Index Copernicus Value (2013): 6.14 | Impact Factor (2015): 6.391}

Further from the Lagrangian density equation (1), now we build a Hamiltonian " $\mathrm{H}$ " that needs to calculate the partition function in term of canonical approach, which is given such as:

$$
\mathrm{Z}(\mathrm{T} . \mathrm{V})=\operatorname{Tr}\left\{\mathrm{e}^{-(\mathrm{H}(\mathcal{L}) / \mathrm{T}}\right\}
$$

Eqn. (2) is to be allowed for all over physical states of the system within a volume V. After that, from the partition function we can obtain all thermodynamic quantities, such as the energy density:

$$
\varepsilon(\mathrm{T})=\lim _{\mathrm{V} \rightarrow \infty}\left\{-\frac{1}{\mathrm{~V}}(\partial \ln Z / \partial(1 / \mathrm{T}))_{\mathrm{V}}\right\}(3)
$$

And the pressure is: $\mathrm{P}(\mathrm{T})=\lim _{\mathrm{V} \rightarrow \infty}\left\{(\partial \operatorname{lnZ} / \partial \mathrm{V})_{T}\right\}(4)$

In general the perturbative calculationslead to the usual divergences of quantum field theory; hence we have to renormalize toobtain finite results, and it cover the entire possible range of behaviour of the system, from confinement to asymptotic freedom, i.e., from nuclear or hadronic matter to ideal chromo-plasma. But this is not applicable due to perturbatively, and we must therefore find a new way to approach the problem, one more generally applicable than the perturbative scheme of quantum electrodynamics (QED). Therefore, the only such approach available up to now is the lattice regularization proposedby Wilson and H. Satz [10, 11 and references therein]. By evaluating the partition function on a large but finite lattice, whose points are separated by multiples of some lattice spacing "a", we have (1/a) aslargest and $1 /(\mathrm{Na})$ as smallest possible momentum; here $\mathrm{Na}$ is the linear size ofthe lattice. Hence neither ultraviolet nor infrared divergences can occur for finiteN and non-zero "a"[11]. We are left with two questions, however: how do we actually carryout the evaluation, and how can we be sure that physical quantities calculated are independentof the lattice? As the functional form of the partition function on thelattice turns out to be quite similar to what one encounters in the evaluation ofspin systems, we can use Monte Carlo simulation techniques $[11,12]$ from statistical physicsto calculate the thermodynamic quantities of interest. Moreover, the renormalization group theory of statistical physics tells us how the lattice spacing "a" and thecoupling factor "g" of the Lagrangian equation (1) [11] must be related, if the results are to be independentof the choice of lattice. So we do indeed have today what seems to be a viableprocedure to evaluate the interesting observables of statistical QCD - and this iscertainly a major reason for the great interest which lattice gauge theory has attractedin recent years[11 and references therein].

\section{Results and Discussions}

Based on above mathematical discussions, we find some results for finite lattice temperature QCD in comparisons to QED. And it is depicted in Table I. In this table, we reported the basic features of QED and QCD. From this table one can concluded that the transition from hadronic matter and/or nuclear matter to a chromo-plasma resembles was such as an insulator-metal transition and in general it is found in the solid state physics [11]. Thus the QCD deconfinement appears same as the metal-insulator transition N. F. Mott [13]. It occurs because of the Debye screenings of a given color charge due to the presence of many other suchcharges [14].
Table 1: The comparison of QCD and QED

\begin{tabular}{|c|c|}
\hline$Q C D$ & $Q E D$ \\
\hline Color charge & Electric charge \\
\hline Hadrons (color neutral) & $\begin{array}{c}\text { Atoms / positronium } \\
\text { (electrically neutral) }\end{array}$ \\
\hline $\begin{array}{c}\text { Hadronic matter } \\
\text { (no color conductivity) }\end{array}$ & $\begin{array}{c}\text { Insulator (no electric } \\
\text { conductivity) }\end{array}$ \\
\hline $\begin{array}{c}\text { Chromo-plasma } \\
\text { (color conductor) }\end{array}$ & Metal (electric conductor) \\
\hline
\end{tabular}

We also used another consequence of the solid state physics, that the conduction electrons in a metal have an "effective" mass different from the electron mass in vacuum, as a collective effect due to the overall field present in the system. In the same way, we suppose that the quarks in the plasma (current quarks with approximate mass, $\mathrm{m}_{\mathrm{q}} \approx 0$ ) to have a different mass than those inside hadrons or hadronic matter (constituent quarks with a mass $m_{q}^{e f f} \approx 200-300 \mathrm{MeV}$ ). The vanishing of the effective quark mass is related to the restoration of the chiral symmetry, which the Lagrangian equation (1) has but which is broken for the actual state of the system at low temperatures. We thus want to study deconfinement and chiral symmetry restoration, as well as the relation of the two phenomena.

Some interesting results have been observed already by various workers [15-20], that in the thermo-dynamical system of the lattice QCD show an important role with dynamical fermions with different types of lattice formulations. Therefore, in Figure 1-3 we have shown some results on such behaviors. In these figures we find a very rapid change in the energy density, $\varepsilon(\mathrm{T})$, with the average thermal loop $\langle\overline{\mathrm{L}}\rangle$ as some ration of confinement and in $\langle\bar{\Psi} \Psi\rangle$ as a measure of chiral symmetry. The unexpected change of all above quantities takes place at the same value of the temperature, which clarify that the deconfinement and chiral symmetry restoration and also occur at the same point. However, some other inferenceis to be drawn in the present investigation that thereis no clear signal for any disappearance of the deconfinement transition with decreasingquark mass, as it was suggested by other works $[21,22]$.

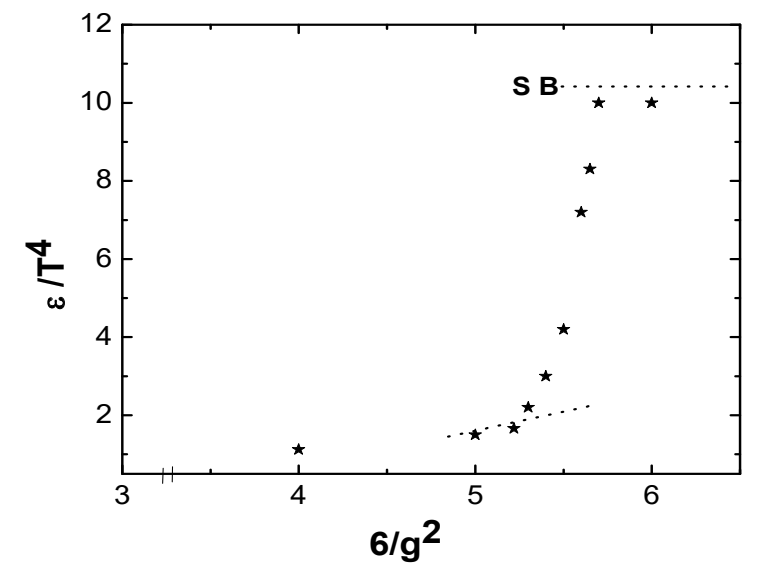

Figure 1: The energy density of quark-gluon system, with fermions, of the order of $\left(8^{2} \times 3\right)$ lattice [ref. 10, 11].

The chiral order parameter for the quark-gluon system, with fermions (canonical) of the lattice order $\left(6^{3} \times 2\right)$ has been 


\section{International Journal of Science and Research (IJSR) \\ ISSN (Online): 2319-7064 \\ Index Copernicus Value (2013): 6.14 | Impact Factor (2015): 6.391}

depicted in Figure 2. These results were found in good agreement with the other workers $[10,11]$.

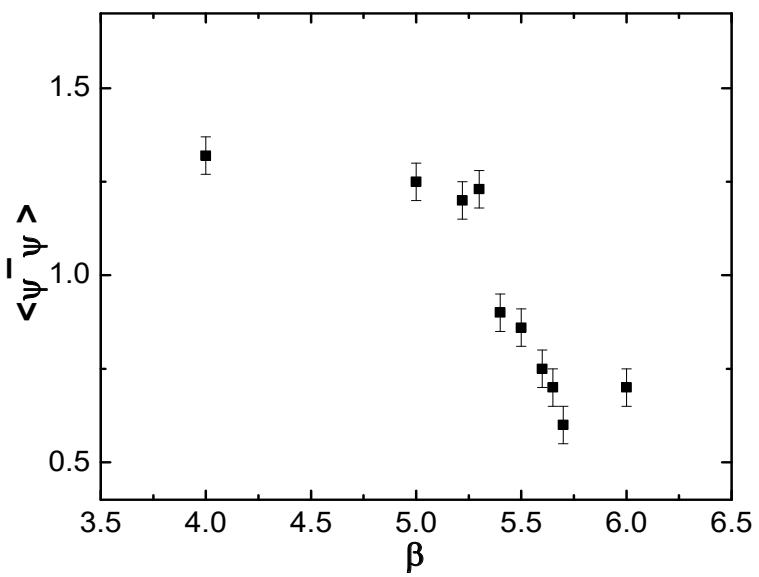

Figure 2: The chiral order parameter for the quark-gluon system, with fermions (canonical) of the lattice order $\left(6^{3} \times 2\right)$ [ref. 10, 11].

Further, we have plotted the average thermal "Wilson loop" $<\mathrm{WL}\rangle$ and the chiral order parameter $\langle\bar{\Psi} \Psi\rangle$ for the quarkgluon system, with fermions (micro-canonical) of the lattice and have been depicted in Figure 3.

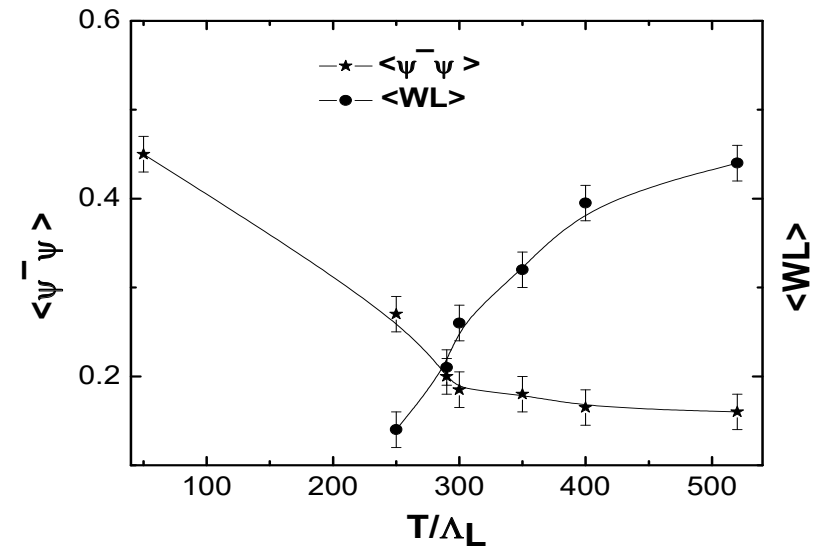

Figure 3: The average thermal "Wilson loop" $<\mathrm{WL}>$ and the chiral order parameter $\langle\bar{\Psi} \Psi\rangle$ for the quark-gluon system, with fermions (micro-canonical) of the lattice [ref. 10, 11].

We indeed for all experimental applications, to find some exact values of the critical temperatures in physical unit for the deconfinement and the chiral symmetry restoration. However, the QCD only predicts some ratios of the physical observables due to no dimensional parameter. Hence we have to calculate the critical temperature $T_{C}$ and some other measured quantity like as $m_{\rho}$ in the lattice units, after that we find a numerical prophecy of $\mathrm{T}_{\mathrm{C}} / \mathrm{m}_{\rho}$. Here the calculations of hadron masses with dynamical fermions have been done properly, so far there is no clear evidencethat the deconfinement temperature differs significantly from the value $\mathrm{T}_{\mathrm{C}} \approx 200 \mathrm{MeV}$ of the pure gluon system. For the existence of a quark-gluon chromo-plasma, this would imply an energy density $\varepsilon_{\text {PLASMA }} \gtrsim 2.5 \mathrm{GeV} / \mathrm{fm}^{3}$.

\section{Conclusions and Final Remark}

On behalf of present research work one can concluded the followings:
We expect that the high energy nuclear collisions will make possible an experimental analysis of strongly interacting matter. On the one hand, this would allow a test of statistical QCD; on the other hand, it provides an empirical simulation of the state of matter of the early universe.

In general, understanding of all said results, it is expected to have philosophical inferences for the "heavy-ion physics", which has been addressing high-temperature QCD with collisions of heavy-ions so far. At the high collider energies the "small reference systems" may also provide testing ground of heavy-ion physics [22].

The transition from quark-gluon plasma to hadronic matter is of the first order, i.e., there should be a coexistence regime, in which the entropy density changes at constant temperature $\left(\mathrm{T}_{\mathrm{C}}\right)$ from its high plasma value to its low hadronic matter value, as a consequence of the expansion of the system. Since the average transverse momentum $\overline{P_{T}}$ of the secondary charged particles give an indication of their emission temperatureand since the multiplicity measures the entropy, this leads to a characteristic relation between $\overline{P_{T}}$ and the average particle multiplicities per central rapidity interval. The high multiplicity cosmic ray events may show such behaviour.

On the other hand, the quark plasma in its initial stage is also in equilibrium with respect to the production of different quark species (chemical equilibrium), then the strange quark content isdetermined at that point. It was first conjectured that this would lead to enhanced strange particle production, and the subsequent considerations [17-20] however, it indicates that expansion effects must be taken into account. Furthermore, these studies, combining simultaneous experimental measurements of soft and hard observables with systematic comparisons to theory, provide the means to reveal the nature and properties of the produced QGP matter.

\section{References}

[1] J. Kapusta, "Quantum Chromo-dynamics at High Tempreture," Nuclear Physics B148, pp. 461-498 (1979).

[2] Shuryak Edward V., "Quantum Chromo-dynamics and the Theory of Superdense Matter", Physics Report 61 pp. 71-158 (1980).

[3] B. Müller: Preprint Duke University, Duke-TH-92-36, Lecture given at NATO ASI on Particle Production in Highly Excited Matter, Ciocco, 1992.

[4] J. Rafelski, "Formation and Observation of the QuarkGluon Plasma," Physics Report 88, No.5, pp. 331-347, (1982).

[5] M. Jacob, J. Tran Thanh Van (eds.), "Quark matter and heavy ion collisions," Physics Report 88, No. 5, 321413, (1982), NORTH-HOLLAND PUBLISHING COMPANY

[6] M. Stephanov, K. Rajagopal and E. Shuryak. "Event-byEvent fluctuations in heavy ion collisions and the QCD crititical point," - art. No. 114028. Physical Review D60 (11) (1999): 4028.

[7] Miklos Gyulassy. "Signatures of new phenomena in ultrarelativistic nuclear collisions," Nuclear Physic, A418, pp. 59-85, (1984). 


\section{International Journal of Science and Research (IJSR) \\ ISSN (Online): 2319-7064 \\ Index Copernicus Value (2013): 6.14 | Impact Factor (2015): 6.391}

[8] J. D. Bjorken. "Highly relativistic nucleus-nucleus collisions: The central rapidity region," Physical Review D27, pp. 140-151, (1983).

[9] W. Marciano and H. Pagels, "Quantum chromodynamics,"Phys. Reports 36 (C), pp. 137-276, (1978).

[10]K. G. Wilson, "Confinement of quarks," Physics Review, D10, pp, 2445-2459, (1974).

[11]H. Satz, "QUARK MATTER 1984: A SUMMARY", Lecture," Lecture Notes in Physics, in Physics, QUARK MATTER Proceedings, pp. 278-297, (1984).

[12] Michael Rreutz, "Monte Carlo study of quantized SU(2) gauge theory," Physics Review, D 21, pp. 2308, (1980).

[13] N. F. Mott, "Metal-Insulator Transition," Rev. Mod. Phys. 40, pp. 677, (1968).

[14] Satz Helmut, "Color Screening in SU(N) Gauge Theory at Finite Temperature, " Nuclear Physics A418, pp. 447C-465C, (1984).

[15] Y. Aoki, G. Endrodi, Z. Fodor, S. Katz, K. Szabo, "The Order of the quantum chromodynamics transition predicted by the standard model of particle physics," Nature 443 pp. 675-678, (2006). arXiv:hep-lat/0611014, doi: 10.1038 /nature 05120

[16]H. Meyer-Ortmanns, T. Reisz, Principles of Phase Structures in Particle Physics, World Scientific Lecture Notes in Physics 77, (2006) 226.

[17]F. Karsch, E. Laermann, A. Peikert, "Quark mass and flavor dependence of the QCD phase transition," Nucl.Phys. B605, pp. 579-599, (2001). doi:10.1016/S0550-3213(01)00200-0.

[18] D. Boyanovsky, H. J. de Vega, D. J. Schwarz, "Phase transitions in the early and the present universe," Ann. Rev. Nucl. Part. Sci. 56, pp.441-500 (2006). doi:10.1146/annurev.nucl.56.080805.140539

[19] K. Adcox, et al., "Formation of dense partonic matter in relativistic nucleus-nucleus collisions at RHIC: Experimental evaluation by the PHENIX collaboration," Nucl. Phys. A757, pp. 184-283, (2005). doi:10.1016/j.nuclphysa.2005.03.086

[20]J. Aichelin, "Quantum molecular dynamics: A Dynamical microscopic $\mathrm{n}$ body approach to investigate fragment formation and the nuclear equation of state in heavy ion collisions," Phys. Rept. 202, 233-360, (1991). doi:10.1016/0370-1573(91)90094-3.

[21]F. Karsch, "Lattice QCD at finite temperature: A Status Report," Z. Physics C: Particles and Fields, Vol. 38, pp. 147-155, (1988). doi:10.1007/BF01574529

[22] Panagiota Foka, Małgorzata Anna Janik, "An overview of experimental results from ultra-relativistic heavy-ion collisions at the CERN LHC: bulk properties and dynamical evolution", Reviews in Physics (2016), doi: 10.1016/j.revip.2016.11.002

\section{Author Profiles}

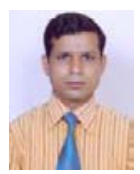

M. Ayaz Ahmad completed Ph.D. in experimental high energy physics in 2010 and M. Phil. (physics) in 2005 from the Physics Department, Aligarh Muslim University, Aligarh, India, under the supervision of Prof. Shafiq Ahmad. He worked as a guest lecturer for B. Sc. Laboratory Classes in the same Department, Aligarh Muslim University, Aligarh w.e.f. 10th Oct. 2002 to 10th Oct. 2008 and also as a Lecturer at Senior Secondary College (Boy's) of Aligarh Muslim University, Aligarh w.e.f. 11th Oct. 2008 to 15th Dec.
2010. Presently, $\mathrm{He}$ is working as an assistant professor at Physics Department, University of Tabuk, Saudi Arabia w.e.f. 16th Dec. 2010. He is involved in teaching and research more than ten years. Besides the undergraduate courses He is teaching/taught courses of Nuclear Physics, Particle Physics and Electrodynamics to graduate / postgraduate students. For the past several years, He is working in the field of Experimental High Energy Heavy Ion Collisions Physics and has published research papers in various refereed journals, like Journal of Physics G (IOP Journal), Nuclear Physics A (Journal of Science Direct/ Elsevier Journals), Journal of Physical Society Japan, Internal National Journal of Mod. Physics E, Ukrainian Journal of Physics, e.t.c.

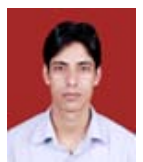

Mir Hashim Rasool completed Ph.D. in experimental high energy physics in 2016 and M. Phil. (physics) in 2012 from the Physics Department, Aligarh Muslim University, Aligarh, India, under the supervision of Prof. Shafiq Ahmad. He has published more than 21 research articles in various National / International Journals. Presently, $\mathrm{He}$ is working as an assistant professor at Department of Physics, Islamic University of Science and Technology, Awantipora, 192122, J\&K, India

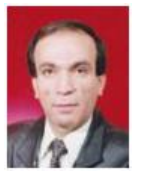

Jalal Hasan Baker completed Ph.D. in experimental nuclear physics in 1996 from the Physics Department, Saint-Petersburg State Technical University, Russia. He is working in Physics Department, Faculty of Science, University of Tabuk, Saudi Arabia since past several years. He has published more than 30 research articles in various National / International Journals.

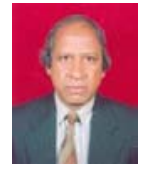

Shafiq Ahmadcompleted Ph. D. in Experimental High Energy Physics in 1980 and M. Phil in 1977 from Aligarh Muslim University, Aligarh. He is working as a Professor at the same University. His working field is relativistic heavy-ion collisions. He is a distinguish personality in his field or academic. He has completed various research projects in collaboration with TIFR and DST, India.

The current objective of his research is to study the existence of dynamical fluctuations. The existence of large fluctuations may also indicate a phase transition. The dynamical fluctuations in the distributions of relativistic shower particles produced in high energy collision may be studied by the method of scaled factorial moments (SFMs). The power law behavior of SFMs is known as Intermittency.

\section{Volume 6 Issue 1, January 2017

